8 th International Symposium on High Energy Spin Physics, Minneapolis, MN, 9/12-17/88.

\section{OPERATION OF THE AGS POLARIZED BEAM*}

Leif A. Ahrens

Brookhaven National Laboratory, Upton, Ni 11973
BNL -41950

DE89 005643

\title{
INTRODUCTION
}

A polarized proton physics run took place during January, 1988, at the Brookhaven AGS. It is the purpose of this paper to review the tune-up period preceding that run. This was the third such run at the AGS, the others occurred in June of 1984 and February of 1986. Some comparisons will be drawn among these. A thorough review of the history and hardware associated with the acceleration of polarized protons at the AGS can be found in the proceedings of the last neeting of this group at Protvinol and will not be repeated here.

THE 1988 RUN

The primary objective for the 1988 run was to obtain a beam suitable for polarized proton physics as rapidly as possible. To maintain polarization 30 imperfection and 5 intrinsic depolarizing resonances hat to be dealt with. After about 2-1/2 weeks of roundthe-clock tuning effort a beam with average polarization of $43 \%$, intensity $1-2 \times 10^{10}$ protons per 2.8 second cycle, at the extraction momentum of $18.5 \mathrm{GeV} / \mathrm{c}$ was achleved. Tuning necessarily continued during the three week physics run which followed. Over this period, the polarization fluctuated from just below $40 \%$ to just above $50 \%$.

The conditions for the 1988 run differed from earlier runs primarily in that the acceleration rate was lower by about a factor of 0.6 due to problems with the main ring magnet power supply. This reduction in crossing speed enhanced all the depolarizing resonances. On the other hand, it resulted in more time between resonances to adjust magnetic corrections. Prior to the ' 88 run a vertical realignment of the naln ring magnets was carried out using a more sophisticated procedure for analyzing the survey data, and taking wore sets of measurements than for similar surveys before the earlier runs. In addition, and unlike previous runs a horizontal realignment was acconplished. Particular attention was paid to centering the ferrite quadrupoles used in jumping the intrinsic resonances on the actual bean equilibriun orbit in both the vertical and horizontal planes.

Before discussing the resonances and their correction, a brief summary will be given of the performance of the other polarized proton acceleration systens. The polarized proton source, which

*Work performed under the ausplces of the U.S. Department of Energy. 
obtalns polarized $\mathrm{H}^{-}$through charge exchange between a neutral cesium beam and a neutral polarized proton beam routinely provided a $500 \mu \mathrm{s}$ pulse of $25 \mu_{\mathrm{A}}$ current. Acceleration to $750 \mathrm{keV}$ was accomplished using an RFO with about a $75 \%$ transmission efficiency. By the time the beam arrived at the $200 \mathrm{MeV}$ end of the Linac, the intensity was about $10 \mathrm{lA}$. At this point, the polarization was periodically monitored by scattering from a carbon target. The polarization was extremely stable at $78 \pm 2 \%$. of the $3 \times 10^{10}$ protons injected into the synchrotron, about $50 \%$ survived to full energy. Capture and acceleration require more sensitive electronic pickups than for normal ( $10^{13}$ protons per pulse) running but that is a solved problen at this intensity level.

A polarimeter located in the main synchrotron ring provides the primary signal for resonance tuning. This "Internal" polarimeter carried a 6 mil dianeter $(0.15 \mathrm{~mm}$ ) nylon fish line this year ( 4 mil in the past). The line both spools ( 1 meter/sec) and flips into the circulating bean when a measurement is desired. The polarization measurenent is derived from the asymmetry measured in two symmetric counter relescopes monttoring the scatter from the string. This yields a relative measure of polarization. The 200 $\mathrm{MeV}$ measure and a measure after extraction from the synchrotron $(18.5 \mathrm{GeV} / \mathrm{c})$ provide two points where the absolute polarization is known.

\section{THE RESONANCES}

The bulk of the effort and the rest of this report will deal with the correcting of the depolarizing resonances. For resonance tuning, the polarization is measured using the internal polarineter on a fixed fleld porch set slightly above the resonance in question. Then all resonances below the porch momentum have been corrected, the porch is moved up in a large enough momentum step to allow a few resonances to be corrected, but not such a large step that the Initial (untuned) polarization on the new porch is negligible. In practice we noved in ten steps from $0.7 \mathrm{GeV} / \mathrm{c}$ to 18.5 $\mathrm{GeV} / \mathrm{c}$ in runing the 35 resonances, taking larger steps in regions known to contain only weak resonances. The resulting plot of polarization at measuring porch against the is a sonewhat distorted saw tooth curve (Figure 1). The maximum polarization gradually declined with increasing momentum, but without clear indication of the structure seen in the past in the region of $G Y=36-y$ and GY $=27$.

The depolarizing resonance condition for the intrinsic resonances is given by $G Y=n P \pm v_{y}$ where $Y=E_{p} / m_{p}$, $G$ is the proton anomalous magnetic moment $(1.7 \%), P$ is the periodicity of the machine (12 for AGS), and $n$ is any integer. The horizontal field driving the spin is just that field responsible for containing the beam vertically in the bean pipe and hence the resonance name. Five such resonant tunes cross the machine tune in accelerating to 
POLARIZATION vS TIME

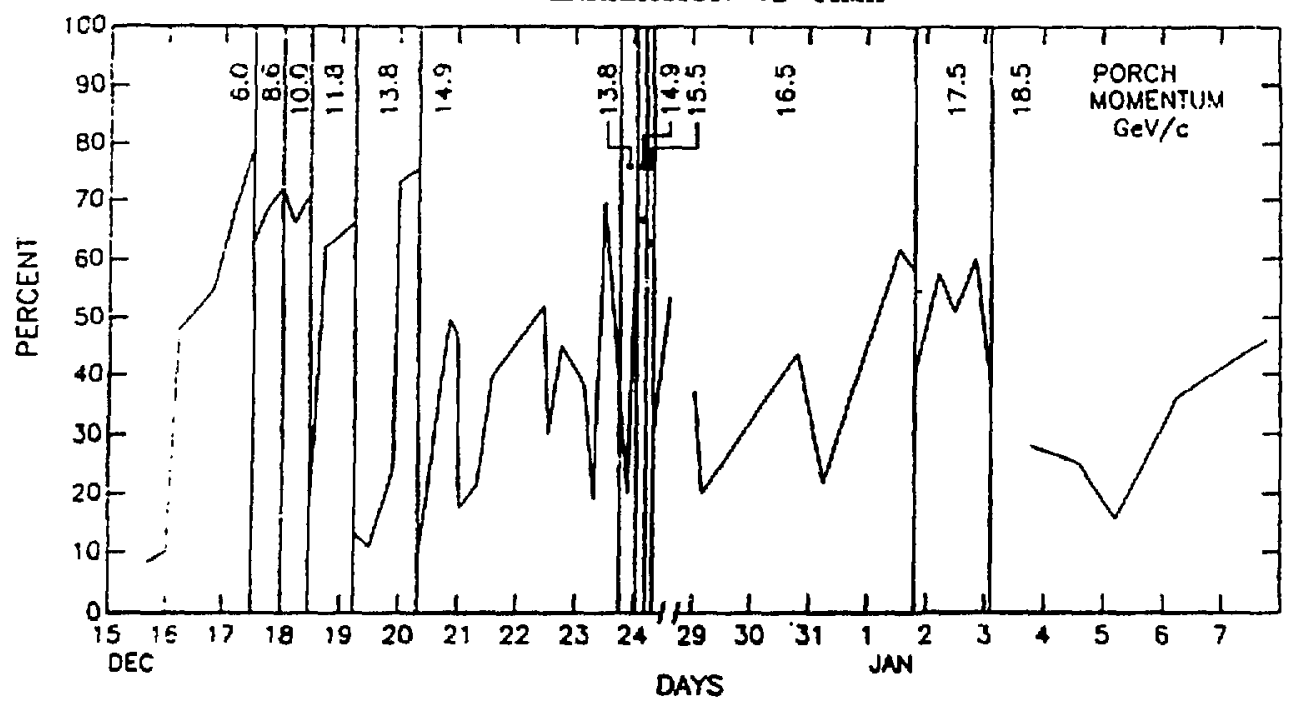

Figure 1. Polarization at the measurement porch momentum as a function of time during the tune up period.

18.5 GeV/c. As an example, we will concentrate on the strongest, at $n=3$, Gr $=36-v_{y}$ or $v_{y}=36-$ Gr. Figure 2 shows this resonance crossing, and the effect of jumping the tune on the rate of

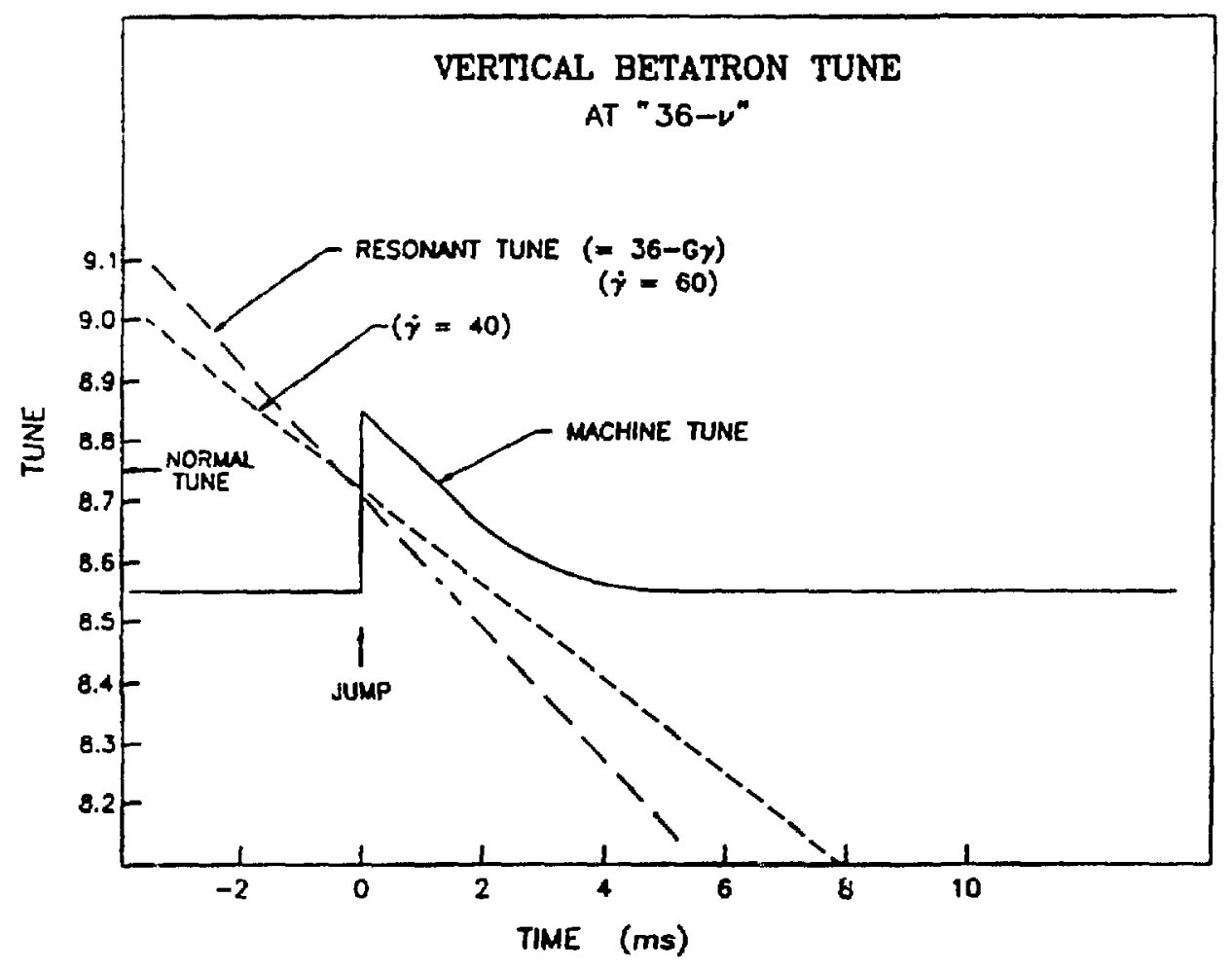

Figure 2. Vertical betatron tune (machine and resonance) vs time. 
crossing. The system which produces the tune jump consists of 10 ferrite core quadrupoles and associated power supplies. What is "tuned" for an intrinsic resonance is the timing of the jump. The clock used ("Gauss" clock) ticks at a rate proportional to the rate of change of magnetic field in the main ring magnets. The optimum timing (or jump field) depends on the machine tune and the particle $\gamma$ (which is uniquely associated with the magnetic field provided the orbit circumference or radius is held fixed). The timing of the tune jump demands more accuracy and stability of the Gauss clock than is required for any other AGS running mode. For the ' 88 run the optimum setting for the jumps occurred within about 10 Gauss of the predicted clock valve. In addition, there was no evidence for a loss of polarization at any intrinsic once the resonance had been tuned. Since the plateau in the curve of final polarization vs jump time is 10 to 20 Gauss wide, these observations imply that the calibration and stability of the Gauss clock over the run was better than one part in one thousand.

The lower acceleration rate for this run mentioned above affected the Intrinsics but only in an indirect way. The actual crossing speed is dominate by the tune jump and so only the distant tails of the resonance are aware of the lower crossing rate. How ever, the curve of $v$ with energy (Figure 2) has a smaller (negative) slope for lower acceleration rate. The wave form for vachine vs time (the fast quad jump and recovery) is cast in concrete (or at least in non-trivial capacitors and resistors) and so could be not changed for the run. The separation between the machine tune and the resonance tune just after the jump was less than for the normal acceleration rate. This pasilem turned out to be important only for $\mathrm{GY}=24+v$ where it showed up as a narrowing of the corrected plateau.

A second problem associated with the intringlc corrections which has plagued us in the past is associated with the very essential non-adiabatic nature of the jump. Although an emittance growth of $10 \%$ per jump is expected from the non-adiabatic shifting of the machine betatron function, in the past ('86) we typically saw increases averaging 50\% per jump and responded by moving the machine tunes around to empirically find the point of minimum growth. As mentioned above, in preparation for the 1988 run, the transverse position of the fast quads was adjusted to center the quads on the orbit. Further, and perhaps more Importantly, we paid particular attention to holding the machine orbit at the centered radius during acceleration. The result of all this was a satisfying reduction in emittance growth (Figure 3) which somewhat reduced the strength of later intrinsic resonances and improved extraction efficlency. Freed of the emittance growth problem the slow adjustwent of the machine tune prior to a jump could be used as original1y designed, namely to increase the jump headroom. By suppressing the nominal 8.75 vertical tune to 8.55 (at $G Y=36$ - v) a jump of 
0.7 units to 8.85 was possible; without the slow adjustment a jump of even 0.2 units caused beam loss from the $v_{v}=9$ stopbands. Slow tune adjustment proved unnecessay at the other incrinsics.

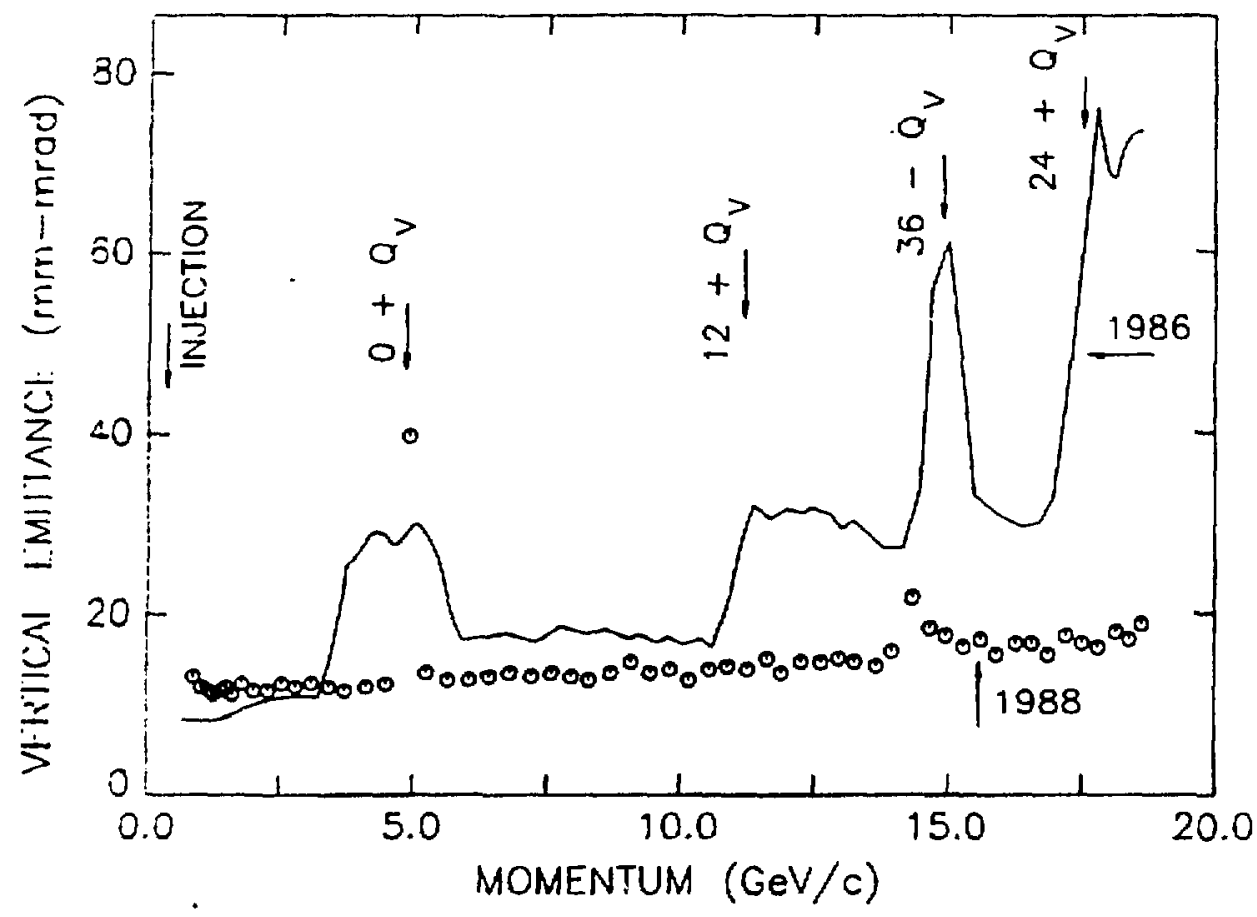

Figure 3. Vertical normalized emittance vs time in the acceleration cycle for ' 86 and ' 88 runs.

Y'inally, the imperfection resonances are discussed. Because there are so many ( 30 below $18.5 \mathrm{GeV} / \mathrm{c}$ ) and all are strong enough to require tuning, imperfection resonance correction is the primary activity of the tune-up period. The depolarizing condition is simply $G r=n$. The resonance is driven by a horizontal magnetic fleld of periodicity $n, B(\theta)=B_{s} \sin (n \theta)+B_{c} \cos (n \theta)$. Tuning Involves adding by means of 95 correction dipoles a magnetic harmonic to cancel the harmonic inftially in the machine. The magnets are energized as the rachine approaches resonance energy with the pulse timing and width set using the Gauss clock calibration to guarantee the resonance is covered.

As in the past imperfection resonances with values of $n=G Y$ near Gr $=36-v(1 . e ., 27,28$, and 29), were more effectively corrected using "beat" harmonics $(9,8$, and 7 respectively) than with the fleld harmonic directly. ${ }^{2}$ These "beat" magnetic harmonics which are integers near 8.75 result in relatively large vertical equilibrium orbit distortions of the machine which carry the 
particles into the horizontal strong focusing fleld of the machine lattice. As a result horlzontal field harmonics at frequencies given by the mixing of the beat harmonic (e.g., 9) and the periodicity ( $\mathrm{K} \times 12 \mathrm{e.g} \cdot, 36)$ of the AGS occur (e.g., 27) which then cause depolarization.

The tune up generates a table of harronic amplitudes which presumably provides a picture of the imperfection harmonics in the machine. It is interesting to compare this table with that generated in previous runs. With the assumption that the imperfection fields come from magnet misalignments, it is approprlate to normalize the corrections to a fixed momentum. Here that momentum is arbitrarily taken. as that for $G Y=7$. A (n) plotted $=A$ (n) measured $x(7 / n)$. Figure 4 compares these amplitudes for the ' 88 and ' 86 run. The few dashed lines for ' 86 were amplitudes measured in the presence of beat corrections and so are not directly comparable. The anplitudes plotted are in units used for the correction. 127 counts corresponded to 10 amps in a magnet located at the peak of the sinusoidal wave. The relative phases for harmonics free of additional beat harmonics is given in Figure 5 . The error bars are derived from the atsumption that the sine and cosine amplitudes corrected the resonance to $0.5 \% .\left(P_{f} / P_{1}=0.995\right)$ There appears to be some correlation between the runs in amplitude and phase. In particular the 12 th harmonic is quite large and reproductble between runs (12 corresponds to the machine pertodicity).

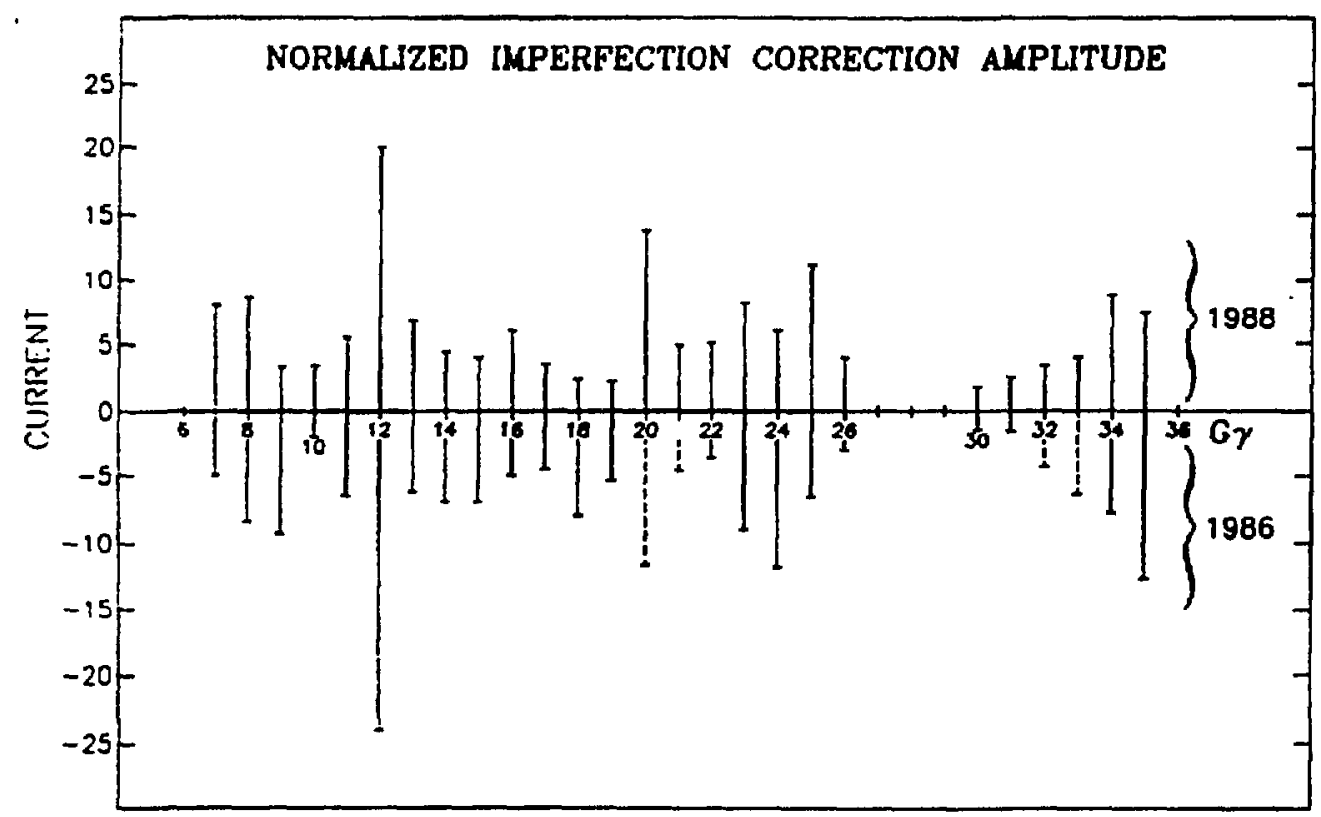

Eigure 4. Harmonic correction amplitudes for the ' 88 and ' 86 runs. (The ' 86 values are plotted with negative sign for comparison.) 


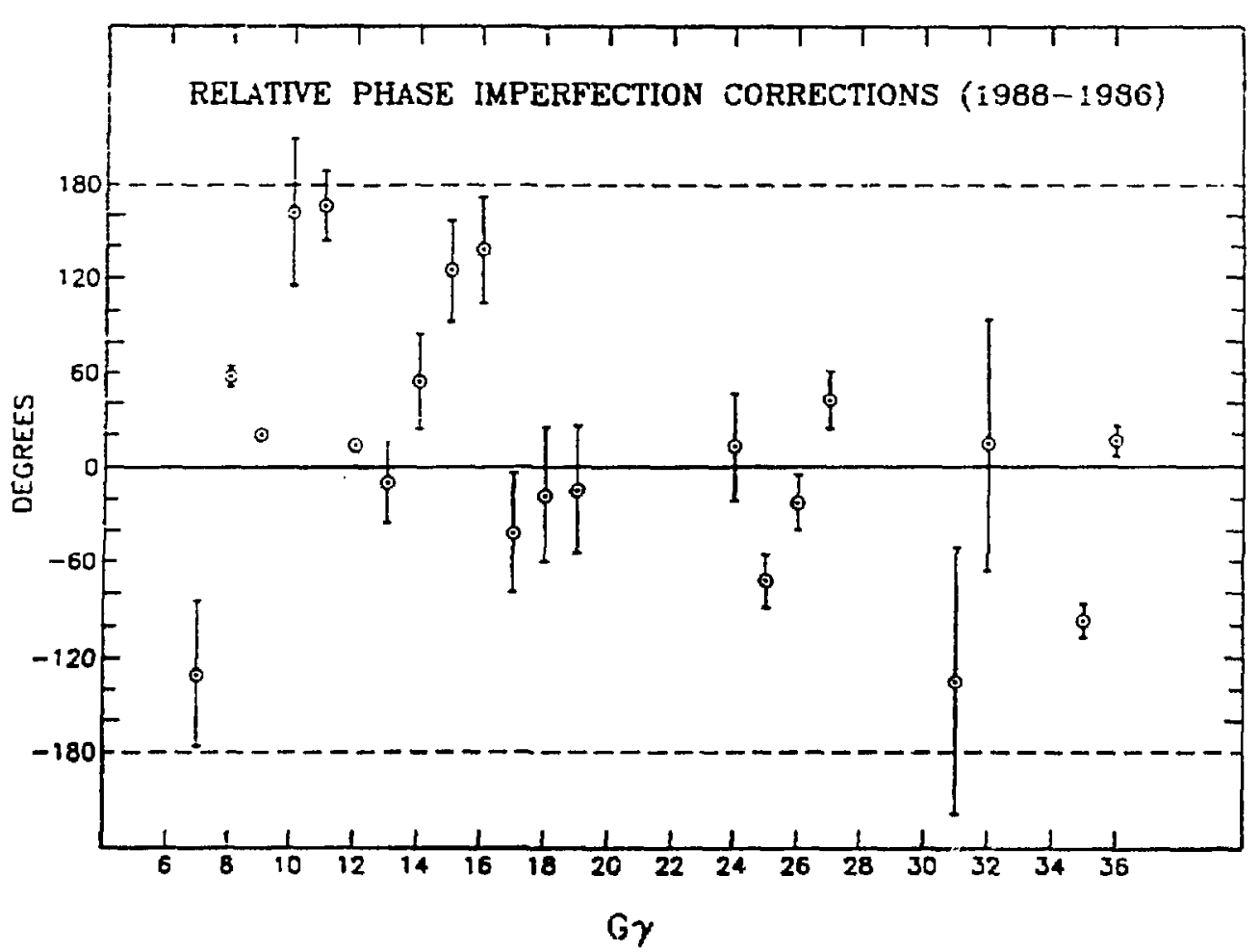

Figure 5. Relative phases of the harmonic corrections between' 88 and ' 86 runs.

The square root of the average of the squares of the amplitudes for 1988, 1986, and 1984 for these harmonics is given in Table I. If one assumes each harmonic is independent, one can ask what rms magnet errors would require such an average magnetic correction. The result is also given in Table I. If the 12 th harmonic is dropped (since it probably results from a systematic problem in the survey) these numbers are reduced by about $1 \mathrm{mil}$. As mentioned above, the magnets have been repositioned prior to each run, with increasing care. For the ' 88 run the rms offset variation was expected to be about $3 \mathrm{mils}$ rather than the $8 \mathrm{mils}$ of Table I. A search for sources of the error field other than vertical misalignment has not yet been productive. The message to the polarization tuner is that a machine which does not require tuning is not likely to result from an improved survey.

For a given resonance, the curve of polarization versus correction amplitude follows the Froissart-Storer formula:

$$
P_{f} / P_{1}=2 \exp \left(-\pi \varepsilon^{2} / \alpha\right)-1,
$$

with strength $\varepsilon$ proportional to the depolarizing field and \& the crossing rate $d \gamma / d \theta$ ( $\theta$ increases by $2 \pi$ in one revolution). As one tunes the depolarizing magnetic harmonic at a resonance one traces out this curve. 
Table I

Ris Values for Correction Harmonic Amplitudes and

Predicted RMS Magnet offsets for the

Three Polarized Proton Runs

\begin{tabular}{c|c|c} 
Run & RMS Mag. Amp & Cal. RMS of fset \\
\hline 1984 & 11.4 & $13.8 \mathrm{mils}$ \\
\hline 1986 & 8.5 & $10.3 \mathrm{mils}$ \\
\hline 1988 & 7.1 & $8.6 \mathrm{mils}$ \\
\hline
\end{tabular}

The full width at half max of the resulting Gaussian gives a figure of merte for the sensitivity of the machine to this harmonic (a small width implies a high sensitivity). Figure 6 gives these widths Eor the 1988 and 1986 runs. The 1988 run was everywhere more sensitive due to the slower acceleration rate. The values near $G Y=9$ are quite different between runs because of the difference in the tune there. (The lack of the need for the slow tune shift to minimize emittance growth mentioned above $\nu_{y} 88 \sim 8.75, \nu_{y}$ $86 \sim 8.5$.

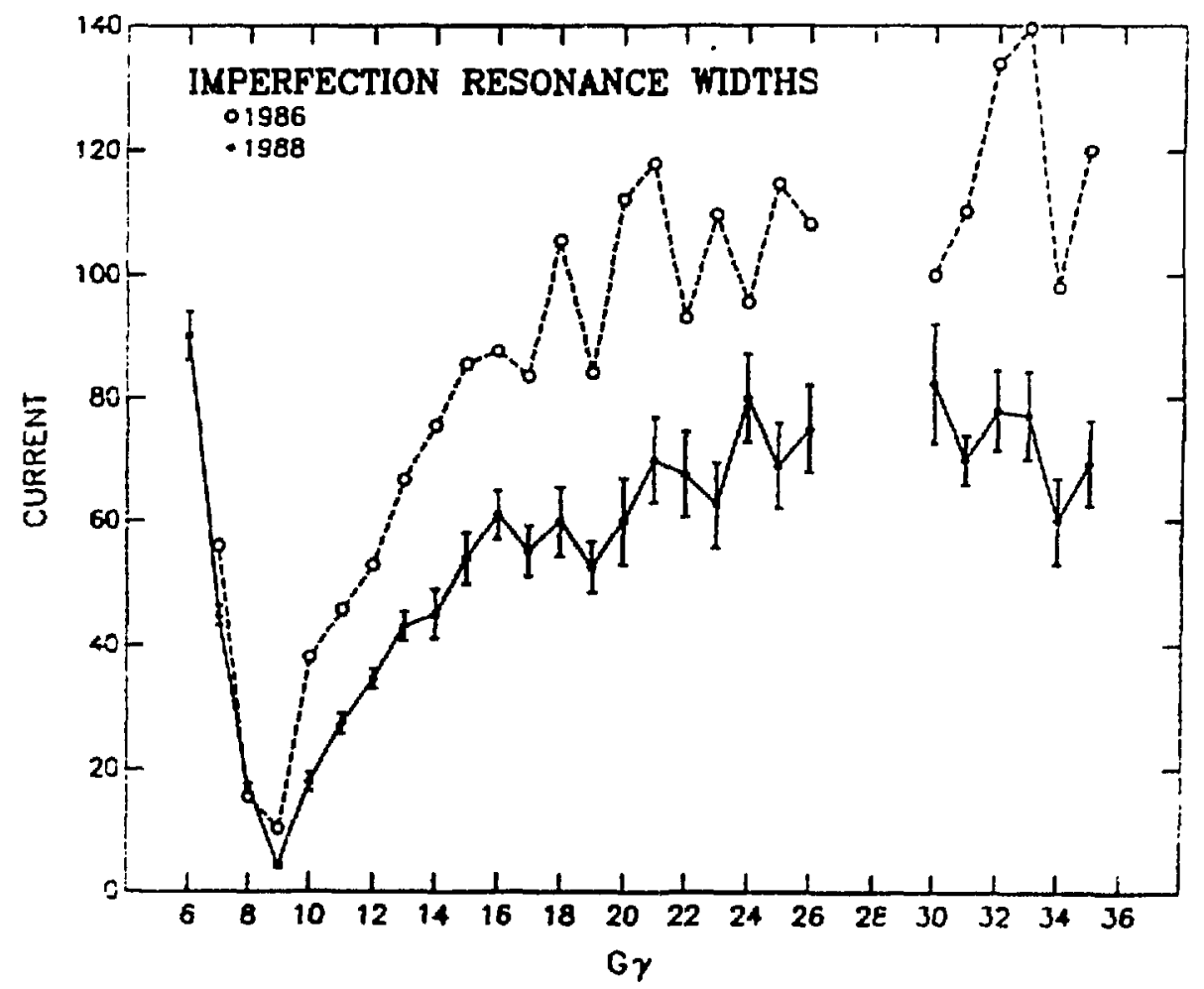

Figure 6. Resonance "widths" vs resonance number. 
Hell into the tune up period, the very sensitive correction at $\mathrm{GY}_{Y}=9$ shifted by a few counts from its corrected value completely destroying the polarization. The cause of the shift, whether magnet notion or a change in the correction system, was not determined but to avoid a repetition of the ordeal it was decided to increase the resonance driving fields enough to completely flip the polarization at $\mathrm{Gr}=9$, gaining a much more stable machine. Flipping was used at three other imperfection resonances during the run in an attempt to further improve stability. The effect on machine performance except at $\mathrm{GY}=9$ was not significant.

\section{CONCLUSIONS}

The 1988 run went closer to the book than earlier runs, the standard techniques worked as advertised. The final polarization at $18.5 \mathrm{GeV} / \mathrm{c}$ was obtained significantly sooner than for past runs, nevertheless, even two weeks of tuning is expensive and an ordeal. The enittance growth was much reduced. The imperfection correction amplitudes required were about the same despite the resurvey. The polarization at $18.5 \mathrm{GeV} / \mathrm{c}$ during the three weeks of physics was not very stable varying from $35 \%$ to $50 \%$. The cause for this frustrating variation was not determined though subtle changes in the magnet positions could cause such an effect acting through the 30 imperfection resonances $\left.(.995)^{30}=.86\right)$. Perhaps we can avoid the problem with a partial snake. (See $T$. Roser, Siberan Snake Workshop Summary, this conference).

\section{ACKNOWLEDGMENTS}

About twenty-four physicists, operation engineers and machine operators took part in the resonance tuning for this beam, a similar number of physicists, engineers and technicians in support groups attended to the special systems need for the run. Their dedicated efforts were essential as was the continuing support from the Iniversity of Michigan Group who participated in the tuning and operated the internal and external polarimeters.

\section{REFERENCES}

1. A.D. Rrisch, Proc. of the 7 th International Symp. on High Enery Spin Physic., Protvino, (1987), P. 41.

2. R.M. Terwlliger et a1., IEEE Trans. on Nucl. Sc1. NS-32, 2635 (1985).

\section{DISCLAIMER}

\footnotetext{
This report was prepared as an account of work sponsored by an agency of the United States Government. Neither the United States Government nor any agency thereof, nor any of their employees, makes any warranty, express or implied, or assumes any legal liability or responsibility for the accuracy, completeness, or usefulness of any information, apparatus, product, or process disclosed, or represents that its use would nol infringe privately owned rights. Refer. ence herein to any specific commercial product, process, or service by trade name, tradenark, manufacturer, or otherwise does not necessarily constitute or imply its endorsement, recommendation, or favoring by the United States Government or any agency thereof. The views and opinions of authors expressed herein do not necessarily state or reflect those of the United States Government or any agency thereof.
} 